\title{
Ultimate Accuracy Limit of Quantum Pulse-Compression Ranging
}

\author{
Quntao Zhuang $\circledast^{1,2, *}$ and Jeffrey H. Shapiro $\odot^{3}$ \\ ${ }^{1}$ Department of Electrical and Computer Engineering, University of Arizona, Tucson, Arizona 85721, USA \\ ${ }^{2}$ James C. Wyant College of Optical Sciences, University of Arizona, Tucson, Arizona 85721, USA \\ ${ }^{3}$ Research Laboratory of Electronics, Massachusetts Institute of Technology, Cambridge, Massachusetts 02139, USA
}

(Received 22 September 2021; accepted 17 December 2021; published 5 January 2022)

\begin{abstract}
Radars use time-of-flight measurement to infer the range to a distant target from its return's round-trip range delay. They typically transmit a high time-bandwidth product waveform and use pulse-compression reception to simultaneously achieve satisfactory range resolution and range accuracy under a peak transmitted-power constraint. Despite the many proposals for quantum radar, none have delineated the ultimate quantum limit on ranging accuracy. We derive that limit through continuous-time quantum analysis and show that quantum illumination ranging - a quantum pulse-compression radar that exploits the entanglement between a high time-bandwidth product transmitted signal pulse and and a high timebandwidth product retained idler pulse-achieves that limit. We also show that quantum illumination ranging offers mean-squared range-delay accuracy that can be tens of $\mathrm{dB}$ better than that of a classical pulse-compression radar of the same pulse bandwidth and transmitted energy.
\end{abstract}

DOI: 10.1103/PhysRevLett.128.010501

Introduction.-Classical microwave radars use time-offlight measurement to infer the range to a distant target from its return's round-trip range delay $\tau$ [1-7]. Their range-delay resolution $\tau_{\text {res }}$, i.e., the delay separation needed for reliably distinguishing between two targets based on their range separation, is inversely proportional to the radar pulse's bandwidth $\Delta \omega$. Their ultimate range-delay measurement accuracy for a single target, i.e., the minimum root-mean-squared (rms) estimation error $\delta \tau_{\min }$ for localizing a single target, as set by the Cramér-Rao bound $(\mathrm{CRB})$, decreases as the signal-to-noise ratio (SNR) increases. A transform-limited transmitted pulse with duration $T$ and peak power $P_{T}$ has $\Delta \omega \sim 2 \pi / T$ and provides an SNR satisfying SNR $\propto P_{T} T$. For a radar whose peak power is constrained, these behaviors lead to a conflict between improving the range-delay resolution and improving the ultimate range-delay accuracy. Using a high time-bandwidth product $(T \Delta \omega \gg 2 \pi)$ transmitted pulse, however, retains the SNR $\propto P_{T} T$ behavior, but pulsecompression reception results in $\tau_{\text {res }} \sim 2 \pi / \Delta \omega \ll T$ [3,4]. Range-delay resolution and CRB accuracy, however, are not the whole story for radar ranging. Because rangedelay estimation is a nonlinear problem, there is a minimum SNR below which range-delay performance is significantly worse than the CRB [8-14]. Here, the Ziv-Zakai bound [8]

Published by the American Physical Society under the terms of the Creative Commons Attribution 4.0 International license. Further distribution of this work must maintain attribution to the author(s) and the published article's title, journal citation, and DOI. affords a useful lower bound on achievable rms accuracy for subthreshold SNRs.

With the emergence of quantum information science, considerable attention is being paid to the notion of quantum radar [15-18]. Much of this work has addressed radar operation at optical wavelengths [19-23], where background noise has low brightness, i.e., $\ll 1$ photon per mode, and very low round-trip radar-to-target-to-radar propagation loss is often assumed. Our interest is in microwave radar, where background noise has high brightness, viz., $\sim 100 \mathrm{~s}-1000 \mathrm{~s}$ photons per mode, and an unresolved target at range $R$ returns a power that is inversely proportional to $R^{4}$, making propagation loss severe. Despite this regime's loss and noise, quantum illumination (QI) [17,24-26] has shown that entanglement offers a $6 \mathrm{~dB}$ advantage over its best classical competitor of the same transmitted energy in the error-probability exponent for detecting the presence of an unresolved target at a particular location.

Recently, QI's hypothesis-testing approach was applied to the task of determining which of many contiguous range-delay resolution bins contains a target that is known to be present in one of them [27]. That first step toward understanding QI's ranging performance did not address QI's ultimate range-delay accuracy, as set by the quantum CRB [28-30] at high SNR and the quantum Ziv-Zakai bound (ZZB) [31] in the subthreshold SNR region. This Letter will remedy those deficiencies by developing a continuous-time framework for QI's entanglement-assisted range-delay estimation and comparing its predictions to corresponding results for classical, i.e., coherent-state, radar. 
Our proposed QI ranging is a quantum pulsecompression radar that benefits from the entanglement between a high time-bandwidth product transmitted signal pulse and a high time-bandwidth product retained idler pulse. In comparison to a classical pulse-compression radar of the same bandwidth and transmitted energy, our quantum CRB analyses show that QI's mean-squared accuracy above its SNR threshold is $3 \mathrm{~dB}$ better than the corresponding above-threshold classical performance. QI's $6 \mathrm{~dB}$ advantage in error-probability exponent over classical radar in determining the target's range-resolution bin [27], however, provides a $6 \mathrm{~dB}$ reduction in its SNR threshold relative to that of classical radar. Remarkably, this threshold reduction translates into an entanglement-assisted meansquared accuracy that can be tens of $\mathrm{dB}$ better than classical performance at the same SNR.

Quantum description of range-delay estimation.-The quantum range-delay estimation problem is as follows. The radar transmits a single spatial-mode field characterized by a photon-units, positive-frequency field operator:

$$
\hat{E}_{S}(t)=\int \frac{d \omega}{2 \pi} \hat{A}_{S}(\omega) e^{-i\left(\omega_{0}+\omega\right) t}
$$

where $\omega_{0}$ is the carrier frequency [32]. In both our classical and quantum pulse-compression radars, this field operator's excitation will have duration $T$, bandwidth $\Delta \omega$ satisfying $2 \pi / T \ll \Delta \omega \ll \omega_{0}$, and average photon number $\mathcal{E}=$ $\int d t\left\langle\hat{E}_{S}^{\dagger}(t) \hat{E}_{S}(t)\right\rangle$. From an unresolved, nonfluctuating target at range $R$, the radar receives a photon-units, positive-frequency field operator $\hat{E}_{R}(t)$ given by

$$
\hat{E}_{R}(t)=\sqrt{\kappa} e^{i \theta_{R}} \hat{E}_{S}(t-\tau)+\sqrt{1-\kappa} \hat{E}_{B}(t),
$$

where $\kappa$ is the round-trip radar-to-target-to-radar transmissivity, $\theta_{R}$ is the phase shift incurred in reflection from the target, $\tau=2 R / c$, with $c$ being light speed, is the target's range delay, and

$$
\hat{E}_{B}(t)=\int \frac{d \omega}{2 \pi} \hat{A}_{B}(\omega) e^{-i\left(\omega+\omega_{0}\right) t}
$$

is the background radiation's field operator. In keeping with a microwave radar's interrogating a distant unresolved target, we shall assume that the target is known to lie in the range uncertainty interval $\mathcal{R}=\left[R_{\min }, R_{\max }\right]$ with $\Delta R \equiv R_{\max }-R_{\min } \ll\left(R_{\min }+R_{\max }\right) / 2$. It then follows that the range-delay $\tau$ will lie in $\left[\tau_{\min }, \tau_{\max }\right]$ with $\tau_{\min }=$ $2 R_{\min } / c, \quad \tau_{\max }=2 R_{\max } / c, \quad$ and $\quad \Delta \tau \equiv \tau_{\max }-\tau_{\min }=$ $2 \Delta R / c$. Also, $\kappa$ will be approximately constant over the range uncertainty interval and satisfy $\kappa \ll 1$. The background radiation-at least over $\hat{E}_{S}(t)$ 's excitation bandwidth - is in a thermal state whose average photon number per mode is $N_{B} /(1-\kappa) \approx N_{B}$, where

$$
N_{B}=1 /\left[\exp \left(\hbar \omega_{0} / k_{B} T_{B}\right)-1\right] \gg 1,
$$

with $\hbar$ being the reduced Planck constant, $k_{B}$ the Boltzmann constant, and $T_{B}$ the radar receiver's noise temperature. The range-delay estimation task is to make a minimum rms error estimate of $\tau$ from a measurement made on $\left\{\hat{E}_{R}(t): t \in \mathcal{T}\right\}$, where $\mathcal{T}$ includes all times for which there could be any target return from the range uncertainty interval.

Before proceeding further, there is an important point to make about $\theta_{R}$ and $\omega_{0}$ that is revealed by the frequency domain version of Eq. (2), viz.,

$$
\hat{A}_{R}(\omega)=\sqrt{\kappa} e^{i\left[\left(\omega_{0}+\omega\right) \tau+\theta_{R}\right]} \hat{A}_{S}(\omega)+\sqrt{1-\kappa} \hat{A}_{B}(\omega) .
$$

If $\theta_{R}$ is modeled as uniformly distributed on $[0,2 \pi]$, as is typically the case, the $\omega_{0} \tau$ term in Eq. (5) becomes uninformative and the radar must implement a measurement that is not destroyed by phase randomness. (In classical radar, this task is accomplished by means of envelope detection after matched filtering.) On the other hand, if $\theta_{R}$ is known, the ensuing range-delay ambiguities spaced $2 \pi / \omega_{0}$ apart [4] prevent the $\omega_{0} \tau$ term in Eq. (5) from being useful. That said, we shall set $\theta_{R}=0$ and $\omega_{0}=0$ in Eq. (5) in evaluating the quantum $\mathrm{CRB}$ and $\mathrm{ZZB}$, recognizing, by convexity, that the results obtained therefrom are lower bounds on their phase-incoherent (random $\theta_{R}$ ) counterparts. See Ref. [33] for evidence supporting the minimal impact, on classical radar, of assuming $\theta_{R}=0$ when $\omega_{0}=0$.

Our rms accuracy $(\delta \tau)$ assessments for the classical and QI pulse-compression radars combine the quantum CRB [28-30] and the quantum ZZB [31]. Specifically, when the radar in question has its SNR above its range-delay estimation's SNR threshold, we use $\delta \tau \approx \delta \tau_{\mathrm{CRB}}=1 / \sqrt{\mathcal{F}_{\tau}}$ [38], where $\mathcal{F}_{\tau}$ is the Fisher information about $\tau$ contained in $\left\{\hat{E}_{R}(t): t \in \mathcal{T}\right\}$ [33]:

$$
\mathcal{F}_{\tau}=\lim _{d \tau \rightarrow 0} \frac{8\left(1-\sqrt{\operatorname{tr}\left[\left(\sqrt{\hat{\rho}_{\tau}} \hat{\rho}_{\tau+\mathrm{d} \tau} \sqrt{\hat{\rho}_{\tau}}\right)^{2}\right]}\right)}{d \tau^{2}},
$$

where $\hat{\rho}_{u}$ is the state of the classical radar's received fieldand, for the quantum radar, the joint state of its received and retained fields-when the range delay is $u$. Alternatively, when the radar's SNR is below threshold, we use $\delta \tau \approx \delta \tau_{\mathrm{ZZB}}$, where

$$
\delta \tau_{\mathrm{ZZB}}=\sqrt{\int_{0}^{\Delta \tau} d \tau^{\prime} \tau^{\prime}\left(1-\frac{\tau^{\prime}}{\Delta \tau}\right) P_{e}\left(\tau^{\prime}\right)},
$$

with $P_{e}\left(\tau^{\prime}\right)$ being the minimum error probability-from the likelihood-ratio test for the classical radar [1] and from the Helstrom limit [28] for the quantum radar-for distinguishing between the equally likely hypotheses $H_{0}=$ target 
present at range delay $\tau_{\min }$ and $H_{1}=$ target present at range delay $\tau_{\min }+\tau^{\prime}$. As will be discussed below, however, we will use the quantum radar's Chernoff bound $[27,39,40]$ in lieu of the Helstrom limit because the former is easily obtained whereas the latter is not [33]. We expect this substitution will have a modest effect on our results because the quantum Chernoff bound is known to be exponentially tight in $P_{e}\left(\tau^{\prime}\right)$ 's SNR dependence.

Classical pulse-compression radar-Our classical pulse-compression radar will emit $\hat{E}_{S}(t)$ in the coherent state $\left|\sqrt{\mathcal{E}} \mathbf{s}(t) e^{-i \omega_{0} t}\right\rangle$, where

$$
\mathbf{s}(t)=\left(2 \pi T^{2}\right)^{-1 / 4} \exp \left(-t^{2} / 4 T^{2}+i \Delta \omega t^{2} / 2 T\right),
$$

with $2 \pi / T \ll \Delta \omega \ll \omega_{0}$. Physically, this is a narrowband but high time-bandwidth product, chirped-Gaussian pulse with average photon number $\mathcal{E}$ and rms time duration $T$. Moreover, because

$$
S(\omega) \equiv \int d t \mathbf{s}(t) e^{-i \omega t} \approx \frac{\exp \left(-\omega^{2} / 4 \Delta \omega^{2}\right)}{\left(\Delta \omega^{2} / 2 \pi\right)^{1 / 4}},
$$

so that $\int(d \omega / 2 \pi) \omega^{2}|S(\omega)|^{2}=\Delta \omega^{2}$, we thus have that $\Delta \omega$ is $\mathbf{s}(t)$ 's rms bandwidth. The quantum CRB-with $\theta_{R}=0$ and $\omega_{0}=0$ - for this classical radar's rms range accuracy with quantum-optimal reception is [33]

$$
\delta \tau_{\mathrm{CRB}}^{\mathrm{C}}=\frac{1}{\Delta \omega \sqrt{2 \kappa \mathcal{E} /\left(N_{B}+1 / 2\right)}} \approx \frac{1}{\Delta \omega \sqrt{2 \mathrm{SNR}}},
$$

where $\mathrm{SNR} \equiv \kappa \mathcal{E} / N_{B}$ and $N_{B} \gg 1$ as is typical for microwave operation.

Interestingly, a semiclassical treatment of a radar using this chirped-Gaussian pulse transmitter and ideal heterodyne reception results in a "classical" CRB that gives the same result as Eq. (10) for $N_{B} \gg 1$. Moreover, for $N_{B} \gg 1$, both the quantum CRB and the classical CRB for heterodyne reception match well-known classical results $[3,4,41-$ 43] for the high-SNR range-delay accuracy. Furthermore, convexity implies that no classical-state transmitter can outperform the best coherent-state radar, and the CRB from Eq. (10) holds for all pulse shapes with rms bandwidth $\Delta \omega$ [33]. Thus, we conclude that a coherent-state transmitter with ideal heterodyne reception is the quantum optimum classical-state radar for above-threshold microwave rangedelay estimation when $\kappa \ll 1$ and $N_{B} \gg 1$.

In contrast to the $\mathrm{CRB}$ - which bounds the range-delay accuracy for estimating an unknown, nonrandom $\tau$-the ZZB is a Bayesian result that assumes $\tau$ to be uniformly distributed on $\tau \in\left[\tau_{\min }, \tau_{\max }\right]$. Consequently, as SNR $\rightarrow 0$, we have $\delta \tau_{\text {ZZB }}$ that will approach the range-delay distribution's standard deviation $\sigma_{\tau}=\sqrt{\Delta \tau^{2} / 12}$, which is typically much greater than the radar's range-delay resolution $\tau_{\text {res }}$. Using well-known results [33]—and $\kappa \ll 1$, $N_{B} \gg 1$-we find that the classical radar's likelihood-ratio test for ideal heterodyne detection results in

$$
\begin{aligned}
P_{e}\left(\tau^{\prime}\right) & =Q\left[\sqrt{\operatorname{SNR}\left(1-e^{-\Delta \omega^{2} \tau^{\prime 2} / 2}\right)}\right] \\
& \leq \exp \left[-\operatorname{SNR}\left(1-e^{-\Delta \omega^{2} \tau^{\prime 2} / 2}\right) / 2\right] / 2,
\end{aligned}
$$

where $Q(x) \equiv \int_{x}^{\infty} d y e^{-y^{2} / 2} / \sqrt{2 \pi}$, and the upper bound is both the classical Chernoff bound for ideal heterodyne reception and the quantum Chernoff bound for the quantum-optimum measurement made on $\left\{\hat{E}_{R}(t): t \in \mathcal{T}\right\}$. The Chernoff bound gives $\delta \tau_{\mathrm{ZZB}-\mathrm{CCB}}^{\mathrm{C}} \sim \sigma_{\tau} e^{-\mathrm{SNR} / 4}$ at low SNRs, but at high SNRs we find [33] $\delta \tau_{\mathrm{ZZB}}^{\mathrm{C}} \rightarrow \delta \tau_{\mathrm{CRB}}^{\mathrm{C}}$ for the exact error probability, whereas $\delta \tau_{\mathrm{ZZB}-\mathrm{C} C \mathrm{C}}^{\mathrm{C}} \rightarrow \sqrt{2} \delta \tau_{\mathrm{CRB}}^{\mathrm{C}}$. The latter behavior is not surprising: at high SNRs, Eq. (7) is dominated by contributions from small values of $\tau$, for which the Chernoff bound is not sufficiently accurate in approximating $P_{e}\left(\tau^{\prime}\right)$ to recover the CRB [33].

Although we have chosen the chirped-Gaussian pulse of Eq. (8) for analytical convenience, the quantum CRB from Eq. (10) and the ZZB's high-SNR and low-SNR asymptotes apply to all pulse shapes with rms bandwidth $\Delta \omega$ [33].

Quantum pulse-compression radar.-Our quantum pulse-compression radar will use a continuous-wave, frequency-degenerate, spontaneous parametric downconverter to produce signal and idler beams whose photon-units, positive-frequency field operators, $\hat{E}_{S}(t)$ from Eq. (1), and

$$
\hat{E}_{I}(t)=\int \frac{d \omega}{2 \pi} \hat{A}_{I}(\omega) e^{-i\left(\omega_{0}-\omega\right) t}
$$

are in a zero-mean jointly Gaussian state characterized by their nonzero Fourier-domain correlations:

$$
\left\langle\hat{A}_{K}^{\dagger}(\omega) \hat{A}_{K}\left(\omega^{\prime}\right)\right\rangle=2 \pi S^{(n)}(\omega) \delta\left(\omega-\omega^{\prime}\right),
$$

for $K=S, I$, and

$$
\left\langle\hat{A}_{S}(\omega) \hat{A}_{I}\left(\omega^{\prime}\right)\right\rangle=2 \pi S^{(p)}(\omega) \delta\left(\omega-\omega^{\prime}\right) .
$$

The quantum radar transmits a $T$-s-long pulse of its signal beam, where $T \gg 2 \pi / \Delta \omega$, while retaining the companion idler pulse for a joint measurement with $\left\{\hat{E}_{R}(t): t \in \mathcal{T}\right\}$.

For analytical convenience, we will take signal and idler's phase-insensitive (fluorescence) spectrum to be

$$
S^{(n)}(\omega) / 2 \pi=\frac{N_{S} e^{-\omega^{2} / 2 \Delta \omega^{2}}}{\sqrt{2 \pi}},
$$

making their average photon number $\int_{0}^{T} d t\left\langle\hat{E}_{S}^{\dagger}(t) \hat{E}_{S}(t)\right\rangle=$ $T \int(d \omega / 2 \pi) S^{(n)}(\omega)=N_{S} \Delta \omega T=\mathcal{E}$ and mean-squared bandwidth $T \int(d \omega / 2 \pi) \omega^{2} S^{(n)}(\omega) / \mathcal{E}=\Delta \omega^{2}$ match that of our classical pulse-compression radar, and we will take the signal and idler's phase-sensitive cross spectrum to be 


$$
S^{(p)}(\omega)=\sqrt{S^{(n)}(\omega)\left(S^{(n)}(\omega)+1\right)},
$$

making their quadratures maximally entangled [17]. Note that the assumption of Gaussian fluorescence spectra is not essential for the quantum advantages derived below to hold [33].

As is well known for QI target detection [17], our quantum pulse-compression radar's performance advantage will come from its phase-sensitive cross spectrum $S^{(p)}(\omega)$, greatly exceeding the classical limit on that cross spectrum $S^{(n)}(\omega)$ when $N_{S} / \sqrt{2 \pi}=\max _{\omega}\left[S^{(n)}(\omega)\right] \ll 1$. This lowbrightness condition implies that our quantum radar will need a much longer pulse duration than a high-brightness classical competitor of the same bandwidth and energy.

The quantum pulse-compression radar's Fisher information is easily evaluated [33], leading-when $N_{S} \ll 1$, $\kappa \ll 1$, and $N_{B} \gg 1$ - to the range-delay CRB

$$
\delta \tau_{\mathrm{CRB}}^{\mathrm{Q}}=1 / 2 \Delta \omega \sqrt{\mathrm{SNR}}=\delta \tau_{\mathrm{CRB}}^{\mathrm{C}} / \sqrt{2},
$$

a result that can be shown to be the best high-SNR performance of all entanglement-assisted radars [33]. When $N_{S} \gg 1$, however, the preceding quantum advantage vanishes, as expected from the previous paragraph, and we get [33] $\delta \tau_{\mathrm{CRB}}^{Q}=\delta \tau_{\mathrm{CRB}}^{C}$.

To find the ZZB for our quantum radar, we will focus on the $N_{S} \ll 1, \kappa \ll 1, N_{B} \gg 1$ regime and use the quantum Chernoff bound (QCB) for range-bin discrimination that applies when $T \gg \Delta \tau[27,33]$, as will be necessary for our quantum radar to reach its threshold SNR, viz.,

$$
P_{e}\left(\tau^{\prime}\right) \leq \exp \left[-2 \operatorname{SNR}\left(1-e^{-\Delta \omega^{2} \tau^{\prime 2} / 2}\right)\right] / 2,
$$

in place of the (challenging to obtain) exact error probability. At high SNRs, we get $\delta \tau_{\mathrm{ZZB}-\mathrm{QCB}}^{Q} \approx \delta \tau_{\mathrm{CRB}}^{Q}$, whereas at low SNRs we find [33] $\delta \tau_{\mathrm{ZZB}-\mathrm{QCB}}^{Q} \approx \sigma_{\tau} e^{-\mathrm{SNR}}$. Comparing to the below-threshold classical result $\delta \tau_{\mathrm{ZZB}-\mathrm{QCB}}^{C} \approx$ $\sigma_{\tau} e^{-\mathrm{SNR} / 4}$ shows the beneficial effect of QI's $6 \mathrm{~dB}$ higher error-probability exponent [24,27].

Strictly speaking, using an error-probability upper bound in an expression that provides a range-delay accuracy lower bound is no longer guaranteed to provide a lower bound. However, because $\delta \tau_{\text {ZZB-QCB }}^{Q}$ approaches the quantum CRB at high SNR and converges to $\sigma_{\tau}$ as SNR $\rightarrow 0$, we believe it to be a good approximation to the $\delta \tau$ of our quantum radar [33].

Accuracy comparison.-Figure 1 presents an example that illustrates the range-delay advantages provided by QI's entanglement-assisted pulse-compression radar over classical pulse-compression radar. There, we plot results for normalized mean-squared range-delay accuracies versus the radar's SNR, both in $\mathrm{dB}$. Here we see that the ZZBs (stars) all show a clear threshold phenomenon, as predicted

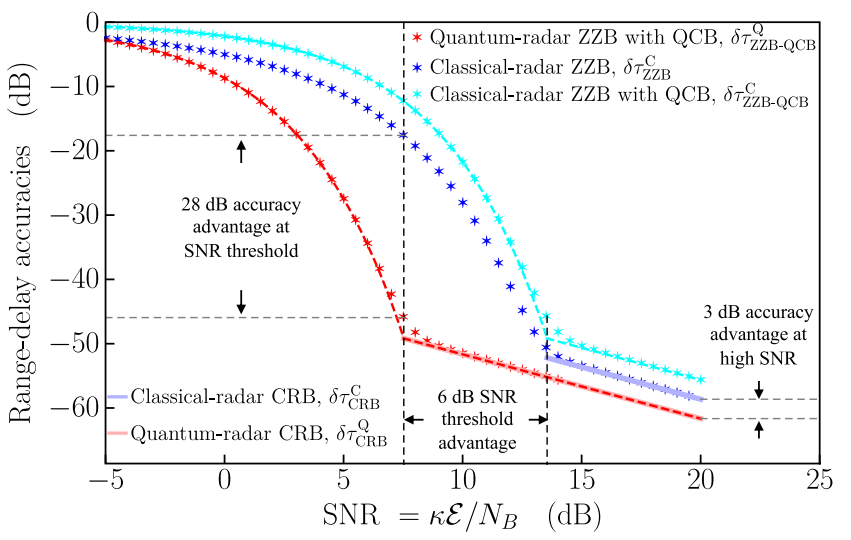

FIG. 1. Normalized mean-squared range-delay accuracies in $\mathrm{dB}, 20 \log _{10}\left(\delta \tau / \sigma_{\tau}\right)$, versus $\mathrm{SNR}$ in $\mathrm{dB}$. The plots assume $\Delta \omega / 2 \pi=10^{6} \mathrm{~Hz}$ and $\Delta R=5 \mathrm{~km}$. Results are shown, top to bottom, for $\delta \tau_{\text {ZZB-QCB }}^{C}$ (cyan stars), $\delta \tau_{\text {ZZB }}^{C}$ (blue stars), and $\delta \tau_{\mathrm{ZZB}-\mathrm{QCB}}^{C}$ (red stars). Also plotted, in the corresponding colors, are their high-SNR and low-SNR asymptotic behaviors, along with vertical dashed lines showing their SNR thresholds, $\mathrm{SNR}_{\text {thresh }}^{C}$ and $\mathrm{SNR}_{\text {thresh }}^{Q}$, computed from the intersections of each radar's high-SNR and low-SNR asymptotic behaviors.

from their high-SNR and low-SNR asymptotic results (dashed lines): $\delta \tau_{\mathrm{CRB}}^{Q}=1 / 2 \Delta \omega \sqrt{\mathrm{SNR}}$ and $\delta \tau_{\mathrm{CRB}}^{C}=$ $1 / \Delta \omega \sqrt{2 \mathrm{SNR}}$ at high SNR, and $\delta \tau_{\mathrm{ZZB}-\mathrm{QCB}}^{Q}=\sigma_{\tau} e^{-\mathrm{SNR}}$ and $\delta \tau_{\mathrm{ZZB}-\mathrm{QCB}}^{C}=\sigma_{\tau} e^{-\mathrm{SNR} / 4}$ at low SNR. For each radar, the threshold signal-to-noise ratio, $\mathrm{SNR}_{\text {thresh }}$, at which range-delay accuracy diverges from the $\mathrm{CRB}$ can be obtained by matching its low-SNR and high-SNR $\delta \tau_{\text {ZZB-QCB }}$ asymptotes. We find that [33]

$$
\mathrm{SNR}_{\text {thresh }}^{Q}=\mathrm{SNR}_{\text {thresh }}^{C} / 4=f\left(1 / 2 \Delta \omega^{2} \sigma_{\tau}^{2}\right) / 2,
$$

where $x=f(y)$ is the inverse function of $y=x e^{-x}$. These thresholds are shown by vertical dashed lines in Fig. 1. They match well to the numerical results (red stars for the quantum radar and cyan or blue stars for the classical radar) found by evaluating Eq. (7). As predicted by Eq. (20), the quantum radar's SNR threshold is $6 \mathrm{~dB}$ lower than that of the classical radar, as highlighted in Fig. 1. This $6 \mathrm{~dB}$ advantage from using entanglement has been verified numerically for a variety of $\Delta \tau$ values [33].

Remarkably, for a $\Delta \omega / 2 \pi=10^{6} \mathrm{~Hz}$ rms-bandwidth quantum radar interrogating a target located within a $\Delta R=$ $5 \mathrm{~km}$ range uncertainty, operation at $\mathrm{SNR}=\mathrm{SNR}_{\text {thresh }}^{Q}$ provides a $28 \mathrm{~dB}$ advantage in mean-squared range-delay accuracy compared to a classical radar of the same bandwidth and pulse energy. More generally, asymptotic analyses [33] show that the quantum radar's mean-squared accuracy advantage when operating at $\mathrm{SNR}_{\text {thresh }}^{Q}$ obeys

$$
\left(\delta \tau_{\mathrm{ZZB}-\mathrm{QCB}}^{C}\right)^{2} /\left(\delta \tau_{\mathrm{ZZB}-\mathrm{QCB}}^{Q}\right)^{2} \sim\left(\Delta \omega \sigma_{\tau}\right)^{3 / 2}
$$




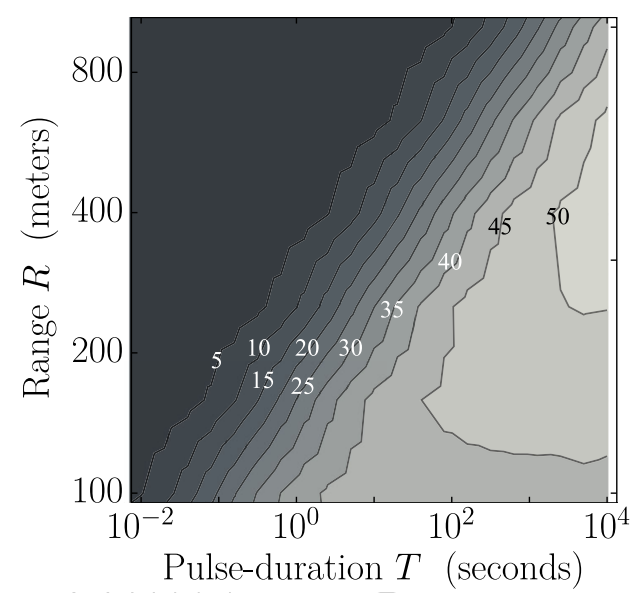

FIG. 2. Contour plot of mean-squared range-delay accuracy advantage (in $\mathrm{dB}$ ) at $\mathrm{SNR}_{\text {thresh }}^{Q}$ assuming range uncertainty $\Delta R=$ $R / 100$ at range $R$.

and thus grows with both increasing bandwidth and rangedelay uncertainty.

Discussion.-Microwave radar is a challenging venue for exploiting quantum entanglement [44]. In this Letter, we developed a continuous-time treatment of QI target ranging and compared its performance to that of a classical pulse-compression radar. Both use time-of-flight measurements to infer target range, hence both have SNR thresholds below which their range-delay measurement accuracy is far worse than their CRB limit. Our quantum radar has a $6 \mathrm{~dB}$ lower threshold SNR than the classical radar. Consequently, when the quantum radar operates at its threshold SNR, its mean-squared range-delay accuracy can be tens of $\mathrm{dB}$ better than that of its classical competitor. Although we have yet to identify a receiver design for achieving our quantum radar's range-delay accuracy advantage, such a large advantage is a much better prospect for retaining a significant advantage with a practical, but suboptimal, receiver than is the case for QI target detection.

Putting aside the task of developing a practical receiver for QI target ranging, any such system will be subject to the same difficulties [17,44-46] that preclude QI's utility for long-range target detection unless exceedingly long pulse durations can be employed so that $\mathrm{SNR}=\kappa M N_{S} / N_{B}$ values-where $M$ is the transmitter's time-bandwidth product [47] —on the order of 5 to $10 \mathrm{~dB}$ can be achieved for $N_{S} \ll 1, \kappa \ll 1$, and $N_{B} \gg 1$.

To illustrate the trade-off between range-delay quantum advantage and pulse duration, consider a $W$-band $\left(\omega_{0} / 2 \pi=100 \mathrm{GHz}\right)$ radar used to localize a tiny unmanned aerial system (UAS). $W$-band radar is both high precision and robust to degraded visual environments [48]; hence, it is widely applicable to UAS detection [49]. Figure 2 is a contour plot of this radar's quantum advantage in mean-squared range-delay accuracy for a $\Delta R=R / 100$ range uncertainty when operated at $\mathrm{SNR}_{\text {thresh }}^{Q}$. It assumes an ideal radar implementation for which $\kappa=\left(G_{T} / 4 \pi R^{2}\right) \times$ $\left(\sigma A_{R} / 4 \pi R^{2}\right)$, with $G_{T}=A_{R} /\left(2 \pi c / \omega_{0}\right)^{2}$ being the radar's antenna gain, $A_{R}=1 \mathrm{~m}^{2}$ its antenna area, and $\sigma=0.01 \mathrm{~m}^{2}$ the UAS's radar cross section. It also assumes a $T_{B}=$ $150 \mathrm{~K}$ noise temperature, giving $N_{B}=32$. These parameters determine the $N_{S}$ needed to reach $\mathrm{SNR}_{\text {thresh }}^{Q}$ and hence the resulting quantum advantage. As seen in Fig. 2, a $0.01 \mathrm{~s}$ pulse duration provides a $20 \mathrm{~dB}$ quantum advantage at $R=100 \mathrm{~m}$, but an impractically long $100 \mathrm{~s}$ pulse duration is needed to realize this same advantage at $R=$ $1 \mathrm{~km}$ because $T / R^{4}$ must be constant to maintain constant quantum advantage.

In conclusion, our Letter's meta lesson is that employing entanglement may offer an enormous performance advantage despite its being used in an entanglement-breaking scenario.

Q.Z. acknowledges the Defense Advanced Research Projects Agency (DARPA) under Young Faculty Award (YFA) Grant No. N660012014029, Office of Naval Research under Grant No. N00014-19-1-2189, Craig M. Berge Dean's Faculty Fellowship of University of Arizona, and support from Raytheon Technologies. J.H.S. acknowledges support from the MITRE Corporation's Quantum Moonshot Program.

*Corresponding author. zhuangquntao@email.arizona.edu

[1] H. L. Van Trees, Detection, Estimation, and Modulation Theory, Part I: Detection, Estimation, and Linear Modulation Theory (Wiley, New York, 2001).

[2] H. L. Van Trees, Detection, Estimation, and Modulation Theory, Part III: Radar-Sonar Signal Processing and Gaussian Signals in Noise (Wiley, New York, 2001).

[3] A. Mallinckrodt and T. Sollenberger, Optimum pulse-time determination, IRE Trans. Inf. Theory 3, 151 (1954).

[4] M. I. Skolnik, Theoretical accuracy of radar measurements, IRE transactions on aeronautical and navigational electronics ANE-7, 123 (1960).

[5] M. I. Skolnik, Introduction to Radar Systems, Third Edition (McGraw-Hill, New York, 2002).

[6] J. Marcum, A statistical theory of target detection by pulsed radar, IRE Trans. Inf. Theory 6, 59 (1960).

[7] Radar Handbook, edited by M. I. Skolnik (McGraw Hill, New York, 2008).

[8] M. Zakai and J. Ziv, On the threshold effect in radar range estimation, IEEE Trans. Inf. Theory 15, 167 (1969).

[9] S.-K. Chow and P. M. Schultheiss, Delay estimation using narrow-band processes, IEEE Trans. Acoust. Speech Signal Process. 29, 478 (1981).

[10] A. Weiss and E. Weinstein, Fundamental limitations in passive time delay estimation-Part I: Narrow-band systems, IEEE Trans. Acoust. Speech Signal Process. 31, 472 (1983).

[11] J. Ianniello, E. Weinstein, and A. J. Weiss, Comparison of the Ziv-Zakai lower bound on time delay estimation with correlator performance, in Proceedings of the IEEE 
International Conference on Acoustics, Speech, and Signal Processing (IEEE, New York, 1983), Vol. 8, pp. 875-878.

[12] E. Weinstein and A. J. Weiss, Fundamental limitations in passive time-delay estimation-Part II: Wide-band systems, IEEE Trans. Acoust. Speech Signal Process. 32, 1064 (1984).

[13] A. Renaux, P. Forster, P. Larzabal, C. D. Richmond, and A. Nehorai, A fresh look at the Bayesian bounds of the WeissWeinstein family, IEEE Trans. Signal Process. 56, 5334 (2008).

[14] D. Dardari, A. Conti, U. Ferner, A. Giorgetti, and M.Z. Win, Ranging with ultrawide bandwidth signals in multipath environments, Proc. IEEE 97, 404 (2009).

[15] M. Lanzagorta, Quantum Radar (Morgan \& Claypool, San Rafael, 2012).

[16] R. G. Torromé, N. B. Bekhti-Winkel, and P. Knott, Introduction to quantum radar, arXiv:2006.14238.

[17] J. H. Shapiro, The quantum illumination story, IEEE Trans. Aerosp. Electron. Syst. 35, 8 (2020).

[18] G. Sorelli, N. Treps, F. Grosshans, and F. Boust, Detecting a target with quantum entanglement, arXiv:2005.07116.

[19] V. Giovannetti, S. Lloyd, and L. Maccone, Quantumenhanced positioning and clock synchronization, Nature (London) 412, 417 (2001).

[20] V. Giovannetti, S. Lloyd, and L. Maccone, Quantumenhanced measurements: Beating the standard quantum limit, Science 306, 1330 (2004).

[21] J. H. Shapiro, Quantum pulse compression laser radar, Proc. SPIE Int. Soc. Opt. Eng. 6603, 660306 (2007).

[22] L. Maccone and C. Ren, Quantum Radar, Phys. Rev. Lett. 124, 200503 (2020).

[23] S. Lloyd, Enhanced sensitivity of photodetection via quantum illumination, Science 321, 1463 (2008).

[24] S.-H. Tan, B. I. Erkmen, V. Giovannetti, S. Guha, S. Lloyd, L. Maccone, S. Pirandola, and J. H. Shapiro, Quantum Illumination with Gaussian States, Phys. Rev. Lett. 101, 253601 (2008).

[25] S. Barzanjeh, S. Guha, C. Weedbrook, D. Vitali, J. H. Shapiro, and S. Pirandalo, Microwave Quantum Illumination, Phys. Rev. Lett. 114, 080503 (2015).

[26] Q. Zhuang, Z. Zhang, and J. H. Shapiro, Optimum MixedState Discrimination for Noisy Entanglement-Enhanced Sensing, Phys. Rev. Lett. 118, 040801 (2017).

[27] Q. Zhuang, Quantum Ranging with Gaussian Entanglement, Phys. Rev. Lett. 126, 240501 (2021).

[28] C. W. Helstrom, Quantum Detection and Estimation Theory (Academic Press, New York, 1976).

[29] H. Yuen and M. Lax, Multiple-parameter quantum estimation and measurement of nonselfadjoint observables, IEEE Trans. Inf. Theory 19, 740 (1973).

[30] A. Holevo, Probabilistic and Statistical Aspects of Quantum Mechanics (North-Holland, Amsterdam, 1982).

[31] M. Tsang, Ziv-Zakai Error Bounds for Quantum Parameter Estimation, Phys. Rev. Lett. 108, 230401 (2012).

[32] The field operators $\hat{E}_{S}(t)$ and $\hat{E}_{S}^{\dagger}(t)$, respectively, annihilate and create photons at time $t$, and their commutator is $\left[\hat{E}_{S}(t), \hat{E}_{S}^{\dagger}\left(t^{\prime}\right)\right]=\delta\left(t-t^{\prime}\right)$, where $\delta(\cdot)$ is the unit impulse. Similarly, their Fourier-domain duals $\hat{A}_{S}(\omega)$ and $\hat{A}_{S}^{\dagger}(\omega)$, respectively, annihilate and create photons at frequency $\omega_{0}+\omega$, and their commutator is $\left[\hat{A}_{S}(\omega), \hat{A}_{S}\left(\omega^{\prime}\right)\right]=2 \pi \delta\left(\omega-\omega^{\prime}\right)$.

[33] See Supplemental Material, which includes Refs. [34-37], at http://link.aps.org/supplemental/10.1103/PhysRevLett .128 .010501 for performance details of our phaseincoherent, classical range-delay estimation, as well as the classical and quantum CRBs and ZZBs for phasecoherent $\omega_{0}=0$ operation.

[34] J. H. Shapiro, The quantum theory of optical communications, IEEE J. Sel. Top. Quantum Electron. 15, 1547 (2009).

[35] H. Scutaru, Fidelity for displaced squeezed thermal states and the oscillator semigroup, J. Phys. A 31, 3659 (1998).

[36] P. Marian and T. A. Marian, Quantum Fisher information on two manifolds of two-mode Gaussian states, Phys. Rev. A 93, 052330 (2016).

[37] C. N. Gagatsos, B. A. Bash, S. Guha, and A. Datta, Bounding the quantum limits of precision for phase estimation with loss and thermal noise, Phys. Rev. A 96, 062306 (2017).

[38] Our radars' high time-bandwidth products guarantee their CRBs' achievability in the high-SNR limit.

[39] K. M. R. Audenaert, J. Calsamiglia, R. MuñozTapia, E. Bagan, L. Masanes, A. Acin, and F. Verstraete, Discriminating States: The Quantum Chernoff Bound, Phys. Rev. Lett. 98, 160501 (2007).

[40] S. Pirandola and S. Lloyd, Computable bounds for the discrimination of Gaussian states, Phys. Rev. A 78, 012331 (2008).

[41] D. Slepian, Estimation of signal parameters in the presence of noise, IRE Trans. Inf. Theory 3, 68 (1954).

[42] V. A. Kotel'nikov, R. A. Silverman, and G. L. Turin, The theory of optimum noise immunity, Phys. Today 13, No. 8 , 40 (1960).

[43] P. M. Woodward, Probability and Information Theory, with Applications to Radar (Elsevier, Amsterdam, 2014).

[44] R. Jonsson and M. Ankel, Quantum radar-What is it good for?, in Proceedings of the 2021 IEEE Radar Conference (IEEE, New York, 2021).

[45] J. H. Shapiro, Microwave quantum radar's alphabet soup: QI, QI-MPA, QCN, QCN-CR, in Proceedings of the 2021 IEEE Radar Conference (IEEE, New York, 2021).

[46] S. Pirandola, B. R. Bardhan, T. Gehring, C. Weedbrook, and S. Lloyd, Advances in photonic quantum sensing, Nat. Photonics, 12, 724 (2018).

[47] For the Gaussian fluorescence spectrum from Eq. (16), we have that $\mathrm{SNR}=\kappa M N_{S} / N_{B}$ with $M=T \Delta \omega$. With this time-bandwidth product, the SNR expression given in the text matches that for the $W-\mathrm{Hz}$ bandwidth, flat fluorescence spectrum- $S^{(n)}(\omega)=N_{S}$ for $|\omega| \leq \pi W$-assumed by Tan et al. [24] and used in many subsequent QI theory papers.

[48] J. N. Sanders-Reed and S. J. Fenley, Visibility in degraded visual environments (dve), Proc. SPIE Int. Soc. Opt. Eng. 10642, 106420S (2018).

[49] M. Caris, W. Johannes, S. Sieger, V. Port, and S. Stanko, Detection of small UAS with W-band radar, in Proceedings of the 18th International Radar Symposium (IEEE, New York, 2017), pp. 1-6. 